OPEN ACCESS

Edited by:

Alain Dervaux,

Centre Hospitalier Sainte-Anne,

France

Reviewed by:

Martin Zack,

Centre for Addiction and Mental

Health, Canada

Kesong $\mathrm{Hu}$,

Cornell University, USA

*Correspondence:

Jasmin Vassileva

jasmin.vassileva@vcuhealth.org

tWoo-Young Ahn and Divya Ramesh contributed equally.

Specialty section:

This article was submitted to Addictive Disorders, a section of the journal

Frontiers in Psychiatry

Received: 06 December 2015 Accepted: 26 February 2016

Published: 10 March 2016

Citation:

Ahn W-Y, Ramesh D, Moeller FG and Vassileva J (2016) Utility of

Machine-Learning Approaches to

Identify Behavioral Markers for Substance Use Disorders: Impulsivity Dimensions as Predictors of Current Cocaine Dependence.

Front. Psychiatry 7:34

doi: 10.3389/fpsyt.2016.00034

\section{Utility of Machine-Learning Approaches to Identify Behavioral Markers for Substance Use Disorders: Impulsivity Dimensions as Predictors of Current Cocaine Dependence}

\author{
Woo-Young Ahn ${ }^{1,2,3 \dagger}$, Divya Ramesh ${ }^{4 \dagger}$, Frederick Gerard Moeller ${ }^{1,2,5}$ and \\ Jasmin Vassileva ${ }^{1,2 *}$
}

'Department of Psychiatry, Virginia Commonwealth University, Richmond, VA, USA, ${ }^{2}$ Institute for Drug and Alcohol Studies, Virginia Commonwealth University, Richmond, VA, USA, ${ }^{3}$ Department of Psychology, The Ohio State University, Columbus, $\mathrm{OH}$, USA, ${ }^{4}$ School of Nursing, University of Connecticut, Storrs, CT, USA, ${ }^{5}$ Department of Pharmacology and Toxicology, Virginia Commonwealth University, Richmond, VA, USA

Background: Identifying objective and accurate markers of cocaine dependence (CD) can innovate its prevention and treatment. Existing evidence suggests that $C D$ is characterized by a wide range of cognitive deficits, most notably by increased impulsivity. Impulsivity is multidimensional and it is unclear which of its various dimensions would have the highest predictive utility for $\mathrm{CD}$. The machine-learning approach is highly promising for discovering predictive markers of disease. Here, we used machine learning to identify multivariate predictive patterns of impulsivity phenotypes that can accurately classify individuals with CD.

Methods: Current cocaine-dependent users $(N=31)$ and healthy controls $(N=23)$ completed the self-report Barratt Impulsiveness Scale-11 and five neurocognitive tasks indexing different dimensions of impulsivity: (1) Immediate Memory Task (IMT), (2) StopSignal Task, (3) Delay-Discounting Task (DDT), (4) lowa Gambling Task (IGT), and (5) Probabilistic Reversal-Learning task. We applied a machine-learning algorithm to all impulsivity measures.

Results: Machine learning accurately classified individuals with $C D$ and predictions were generalizable to new samples (area under the curve of the receiver-operating characteristic curve was 0.912 in the test set). CD membership was predicted by higher scores on motor and non-planning trait impulsivity, poor response inhibition, and discriminability on the IMT, higher delay discounting on the DDT, and poor decision making on the IGT.

Conclusion: Our results suggest that multivariate behavioral impulsivity phenotypes can predict CD with high degree of accuracy, which can potentially be used to assess individuals' vulnerability to $C D$ in clinical settings.

Keywords: cocaine, addiction, substance dependence, impulsivity, machine learning, LASSO 


\section{INTRODUCTION}

Substance misuse is one of the biggest public health problems that have a major impact on our societies and nations. The total cost of substance use disorders (SUDs) is estimated at over $\$ 500$ billion a year in the United States (1). Unfortunately, efficacious pharmacological and behavioral interventions are limited for most SUDs [but see Ref. (2)]: some medications exist for tobacco- and opiate dependence, but none are available for cocaine-, methamphetamine-, or cannabis dependence (3).

One of the key features of SUDs is impulsivity, defined as "a predisposition toward rapid, unplanned reactions to internal or external stimuli with diminished regard to the negative consequences of these reactions to the impulsive individual or to others." $(4,5)$. Impulsivity is a multidimensional construct manifested in various ways $(5,6)$. Most often, impulsivity is measured by self-report measures of trait impulsivity, which assess impulsivity as a long-lasting personality characteristic. Impulsivity is also indexed by laboratory neurocognitive measures that most commonly assess two main processes: (1) impulsive action (7), i.e., compromised ability to inhibit inappropriate behaviors, and (2) impulsive choice (8), reflecting suboptimal choices in the face of delay contingencies or potential reward/risk. Note that these two constructs are classified differently in value-based decision-making literature (e.g., action selection and valuation systems, respectively) (9). Importantly, these various dimensions of impulsivity often do not correlate to each other (10-13), suggesting that self-report and neurocognitive tasks of impulsivity reflect different processes (14).

Previous studies show that different dimensions of impulsivity are strong predictors of drug initiation and maintenance (15-18) and are also associated with clinical treatment outcomes, such as poor treatment response and increased propensity for relapse [e.g., Ref. (19)]. Cocaine dependence (CD), among other SUDs, is strongly related to increased impulsivity (20). Cocainedependent individuals (CDIs) score consistently higher than healthy controls (HCs) on self-report trait impulsivity measures $(19,21,22)$. Numerous neurocognitive studies also demonstrate that CDIs are characterized by both impulsive action and impulsive choice. With regards to impulsive choice, CDIs discount delayed rewards more steeply compared to HCs $(23,24)$; show decision-making deficits on gambling tasks, such as the Iowa Gambling task (IGT) $(11,25)$; and have difficulties adaptively reversing their choice preference, evidenced by increased perseverative errors on Probabilistic Reversal-Learning (PRL) tasks (26). On measures of impulsive action, CDIs show impaired response inhibition compared to HCs on neurocognitive tasks, such as the Immediate Memory Task (IMT) $(21,22)$ and the Stop-Signal Task (SST) $(27,28)$.

These results suggest that a multivariate battery of multiple behavioral measures could more accurately predict cocaine dependence compared to single (i.e., univariate) measures. The discovery of strong and generalizable markers of CD could help us more objectively diagnose $\mathrm{CD}$ and lead to important innovations in clinical settings, such as personalized prevention and intervention programs. Clearly, identifying predictors or classifiers of CD and more generally of SUDs would be a useful aim, but the field is still in its infancy (29). One of the most promising approaches is the application of machine-learning methods. Unlike conventional univariate methods, which compare healthy and psychiatric groups on each measure at a time, machine-learning (supervised learning) methods select multivariate predictive patterns of data in a way that optimizes prediction accuracy in new samples (29-31). A machinelearning approach is particularly useful when there are a large number of predictor variables (i.e., the dimensionality of feature space is large) compared to the number of samples or when our focus is on the reliability and generalizability of measures (30). However, to our knowledge, few studies have investigated SUDs from a machine-learning perspective $(32,33)$ and no studies have employed multiple dimensions of impulsivity as predictors in a machine-learning model.

We address these gaps by using the machine-learning method and a battery of behavioral measures assessing various aspects of trait impulsivity, impulsive choice, and impulsive action. The main goal of the study was to identify multivariate patterns of impulsivity that (1) best predict CD and (2) make accurate predictions that are generalizable to new samples. Here, we demonstrate that a machine-learning algorithm can identify multivariate impulsivity profiles that predict $\mathrm{CD}$ in new samples with high degree of accuracy.

\section{MATERIALS AND METHODS}

\section{Participants}

The sample consisted of $23 \mathrm{HCs}$ and $31 \mathrm{CDIs}$ recruited from ongoing studies at the Institute for Drug and Alcohol Studies (IDAS) at Virginia Commonwealth University (VCU). Participants were recruited via newspaper advertisements and were initially screened by a brief telephone interview. Individuals were excluded if they indicated significant psychiatric or medical conditions, including a self-reported history of severe brain injury. Following the phone screen, eligible participants attended an in-person intake assessment session, where they were screened for psychiatric disorders using the structured clinical interview for DSM-IV (SCID-I) (34), and completed a medical history and physical examination. Information about the participants' demographic and drug use history was also collected at the intake interview. All participants were urine tested for cocaine (benzoylecgonine), tetrahydrocannabinol (THC), opiates, amphetamine, methamphetamine, and benzodiazepines using integrated E-Z split key cup II (Innovacon Company, San Diego, CA, USA) on each visit to the clinic. Eligible cocaine-dependent participants met current DSM-IV criteria for CD; did not meet DSM-IV current dependence criteria for drugs other than cocaine, marijuana, nicotine, or alcohol; and did not have current or past medical disorders affecting the central nervous system. The cocaine-dependent sample included both treatment-seeking $(n=22)$ and non-treatmentseeking $(n=9)$ participants. The treatment seekers were part of studies, in which they received manualized cognitive behavioral therapy and were randomized to either placebo or any one or combination of the following medications: levodopa/carbidopa and/or citalopram. All data from treatment seekers were collected 
at intake prior to the start of medication or behavioral therapy; therefore, treatment seekers and non-treatment seekers have been grouped together for the analyses.

The HC group consisted of participants who had negative urine drug screen, negative breathalyzer test, and did not have any current or past DSM-IV axis I disorders (including substance dependence) or medical disorders affecting the central nervous system. HCs were recruited via similar advertising procedures as the cocaine-dependent participants.

All participants were free of alcohol at the time of testing as determined by a breathalyzer (Intoximeters, Inc., St. Louis, MO, USA). Female participants were excluded if they had a positive urine pregnancy test. All data were collected at the VCU IDAS. All participants were compensated for their participation. Participants were fully informed of the nature of the research and provided written consent for their involvement in accordance with the Declaration of Helsinki. The studies from which subject data were included were approved by the Committee for the Protection of Human Participants at VCU.

\section{Impulsivity Measures \\ Trait Impulsivity \\ Barratt Impulsiveness Scale-11}

The Barratt Impulsiveness Scale-11 (BIS-11) is one of the most commonly used measures of trait impulsivity. The BIS-11 is a 30-item self-report scale with three oblique factors: (1) attentional/ cognitive impulsivity, measuring tolerance for cognitive complexity and persistence; (2) motor impulsivity, measuring the tendency to act on the spur of the moment; and (3) non-planning impulsivity, measuring the lack of sense of the future. Total scores range from 30 to 120, with non-psychiatric controls generally scoring 50-60 $(35,36)$.

\section{Impulsive Action}

\section{Immediate Memory Task}

The IMT is a more complex version of the continuous performance test (CPT) designed to measure sustained attention, working memory, and response inhibition. The IMT consists of two blocks of 180 trials each. In each trial, a series of five-digit numbers (e.g., 73021) are displayed on the monitor for $0.5 \mathrm{~s}$ and separated by a 0.5 -s interstimulus interval. Participants are instructed to respond when a five-digit number (the target stimulus) is identical to the preceding stimulus. The probability of a target stimulus is set at $33 \%$. Three types of stimuli are presented: target (33\%), catch (33\%), and filler (34\%). A target stimulus is a five-digit number that is identical to the preceding number. Responses to target stimuli are recorded as correct detections (or "hits"), whereas a failure to respond to a target stimulus is recorded as an omission error (or "false-negative"). A catch stimulus is a number that differs from the preceding number by only one digit (position and value determined randomly). Responses to catch stimuli are recorded as commission errors (or "false positives"). A filler stimulus is a random five-digit number that appears whenever a target or catch trial is not scheduled to appear. Responses to filler stimuli are recorded as random errors. We used four indices of impulsivity from the IMT: non-parametric discriminability $\left(\mathrm{A}^{\prime}\right)$ that does not require the underlying distributions to be normally distributed, response bias $\left(\mathrm{B}_{\mathrm{D}}\right)(22)$ derived from signal detection theory (38), commission errors, and omission errors (37).

\section{Stop-Signal Task}

The SST measures motor impulsivity/impulsive action, defined as the inability to inhibit an already triggered motor response. In this task, participants are required to make quick key responses to visually presented "go" signals and to inhibit any response when an auditory stop signal is suddenly presented. The task consists of two phases: a practice phase of 32 trials and an experimental phase of three blocks of 64 trials per block. The primary task is a shape judgment task that requires participants to discriminate between a square and a circle. In no-signal trials (75\% of the trials), only the primary-task stimulus is presented, and participants are instructed to respond to the stimulus as fast and accurately as possible. Occasionally (25\% of the trials), a stop signal $(750 \mathrm{~Hz}$, $75 \mathrm{~ms}$ ) is presented shortly after the stimulus onset in the primary task. In the stop-signal trials, the primary-task stimulus is followed by the auditory stop signal presented after a variable stopsignal delay (SSD), and participants are instructed to withhold their responses. The SSD is initially set at $250 \mathrm{~ms}$ and is adjusted continuously with the staircase tracking procedure: when inhibition is successful, SSD increases by $50 \mathrm{~ms}$ and when inhibition is unsuccessful, SSD decreases by $50 \mathrm{~ms}$. The index of impulsivity of the SST is stop-signal reaction time (SSRT), which is the speed of the inhibitory process. SSRT was estimated by subtracting average SSD of the three blocks from median no-signal reaction time $(39,40)$.

\section{Impulsive Choice \\ Adjusting Delay-Discounting Task}

This task is designed to measure participants' discounting rate when they are presented with the possibility of receiving a hypothetical reward determined using a choice algorithm. The task is presented on a computer screen displaying two large command buttons, one on the left and the other on the right side of the screen. The left button always displays an immediate adjusting reward (e.g., "\$5.00 now"), and the right button displays a delayed reward (e.g., "\$10.00 in 1 week"). Participants are exposed to a series of choices where the delay reward magnitudes are $\$ 10, \$ 25$, $\$ 100, \$ 250, \$ 1,000$, or $\$ 2,500$ at delay periods of 1 day, 1 week, 1 month, 6 months, 1 year, 5 years, or 25 years. The computer program varies the smaller, immediately available amounts across trials according to the algorithm. However, the larger delayed amount stays the same until an indifference point (i.e., where subjective values of immediate and delayed rewards are equivalent) is determined. After an indifference point is determined, the delay for the larger reward increases to the next duration. Participants are randomly assigned to complete the assessment in either ascending or descending order of delays. Non-linear regression was used to fit a hyperbolic function that has a single free parameter, $k$ (discounting rate) and natural logarithm of transformation, $\log (k)$, was used to normalize the distribution of $k$ across participants $(23,41)$. 
The Kirby Monetary-ChoiceDelay-DiscountingQuestionnaire Participants are presented with a fixed set of 27 choices between smaller, immediate rewards (SIRs) and larger, delayed rewards (LDRs). For example, in the first trial, participants are asked, "Would you prefer $\$ 54$ today or $\$ 55$ in 117 days?" The participant indicates which alternative s/he would prefer by circling it on the questionnaire. The order is contrived such that the trial order does not correlate to the SIR or LDR amounts, their ratio, their difference, the delay to the LDR, or the discount rate corresponding to indifference between the two rewards. An estimate of a participant's $\log (k)$ was made from the participant's pattern of choices across the 27 questions on the monetary-choice questionnaire and by fitting a hyperbolic function with logistic regression $(42,43)$.

\section{The Iowa Gambling Task}

In this computerized version of the task, participants are asked to choose between four decks of cards that result in hypothetical monetary rewards, with the goal to maximize gains. Each deck (labeled A-D) contains 60 cards. Participants must make 100 choices over the testing session. Two of the decks (A and B) are disadvantageous in that they are associated with high immediate rewards but even higher subsequent losses. Decks C and D are considered advantageous because they result in an overall long-term gain. The index of decision-making performance on the IGT is a "net score" based on the total number of cards selected from the advantageous minus the disadvantageous decks $[(C+D)-(A+B)]$ across the 100 trials. Lower net score indicate less advantageous decision making (44).

\section{The Probabilistic Reversal-Learning Task}

In the Reversal-Learning task, participants are required to choose between two stimuli that differ in color. One stimulus is "correct" and the other one is "wrong," and participants have to discover which of the two cards is correct using trial-by-trial experience and are instructed to win as much money as possible. Selection of the correct or wrong stimulus leads to positive feedback 80 or $20 \%$ of the trials, respectively. After 40 trials (stage 1), the contingencies for correct and incorrect stimuli are reversed for the subsequent 40 trials (stage 2 ). In other words, the correct stimulus in stage 1 becomes an incorrect stimulus in stage 2 , and vice versa for incorrect stimulus. Only participants who pass stage 1 (eight consecutive correct responses) are included in the analysis. The ability to reverse the acquired stimulus-reward association is measured by the number of consecutive responses to the incorrect stimulus immediately following the reversal in contingencies (perseverative errors), which was uniquely impaired among chronic cocaine users in a previous study $(26,45)$.

\section{Machine-Learning Approach}

To identify multivariate profiles that correctly classify and distinguish CDIs from HCs, we applied a machine-learning algorithm called the "least absolute shrinkage and selection operator" [LASSO (46)] to self-report and neurocognitive measures of impulsivity and demographic variables. Our goal was to find the precise combination of impulsivity indices that contains the minimal number of measures for the shortest testing duration, yet provides comprehensive evaluation of impulsivity and accurate group classification. The LASSO is a penalized regression method, which imposes L1 penalty indicating that the sum of absolute values of coefficients is constrained. The LASSO automatically selects variables that are important for predictions and shrinks the coefficients of unimportant variables toward 0 , which improves the prediction accuracy of the regression model to new samples. We chose the LASSO in this study because it searches for the most parsimonious model compared to other penalized regression methods. Note that unlike our approach, traditional multiple regression methods are unable to select variables and fitted models are more suceptible to over-fitting.

With the exception of the LASSO and fivefold cross-validation $(\mathrm{CV})$, the core procedures for generating out-of-sample prediction (penalized logistic regression) are identical to those used in a previous study (47), which provides detailed illustration of the method and step-by-step procedures. The dependent variable we aimed to classify was whether an individual meets criteria for CD or not. For computing out-of-sample predictions, we randomly split the whole dataset into a training set (67\% of the data) and a test (validation) set ( $33 \%$ of the data). After splitting the dataset into the training ( $67 \%$ of the data) and test $(33 \%$ of the data) sets, we fitted the LASSO model that minimized binomial deviance in the training set using 1,000 iterations of fivefold CV (i.e., divided the training set into five partitions, trained the LASSO using only four partitions, tested the model on the remaining 1 partition, and repeat the $\mathrm{CV}$ procedure five times, with each of the five partitions used only once as the validation data) $(32,47)$. Then, we made predictions on the test set based on the LASSO model estimated only with the training set. We also report prediction performance of the model on the training set for completeness.

For identifying predictors of cocaine dependence, we used fivefold CV across all samples, in which we used the same data as the training set and the test set. The goal was to identify predictors that are most robust across all samples. Alternatively, we could divide data into the training and test sets, identify beta coefficients of survived predictors in the training set, repeat the procedure for each randomly selected training set, and average the beta coefficients over all repetitions. Estimated beta coefficients remain essentially the same for both approaches. The mean beta coefficients over 1,000 iterations were plotted in Figure 1 where we set the mean beta coefficients of measures that survived less than $5 \%$ of 1,000 iterations to 0 for visualization purposes (their actual values are close to 0 anyway).

For the estimation of the LASSO model, we used the glmnet package (48). Model performance was indexed using the area under the curve (AUC) of the receiver-operating characteristic (ROC) curve, which quantifies the ability of the model to correctly assign a participant to the $\mathrm{CD}$ group. The $\mathrm{pROC}$ package (49) was used for generating a ROC curve and computing the AUC. A perfect model and a random model will score an AUC of 1.0 and 0.5 , respectively. AUC values between 0.9 and 1 are considered outstanding discrimination and values between 0.8 and 0.9 are considered excellent (50). Note that in each of 1,000 iterations, we computed each individual's "response" or fitted 


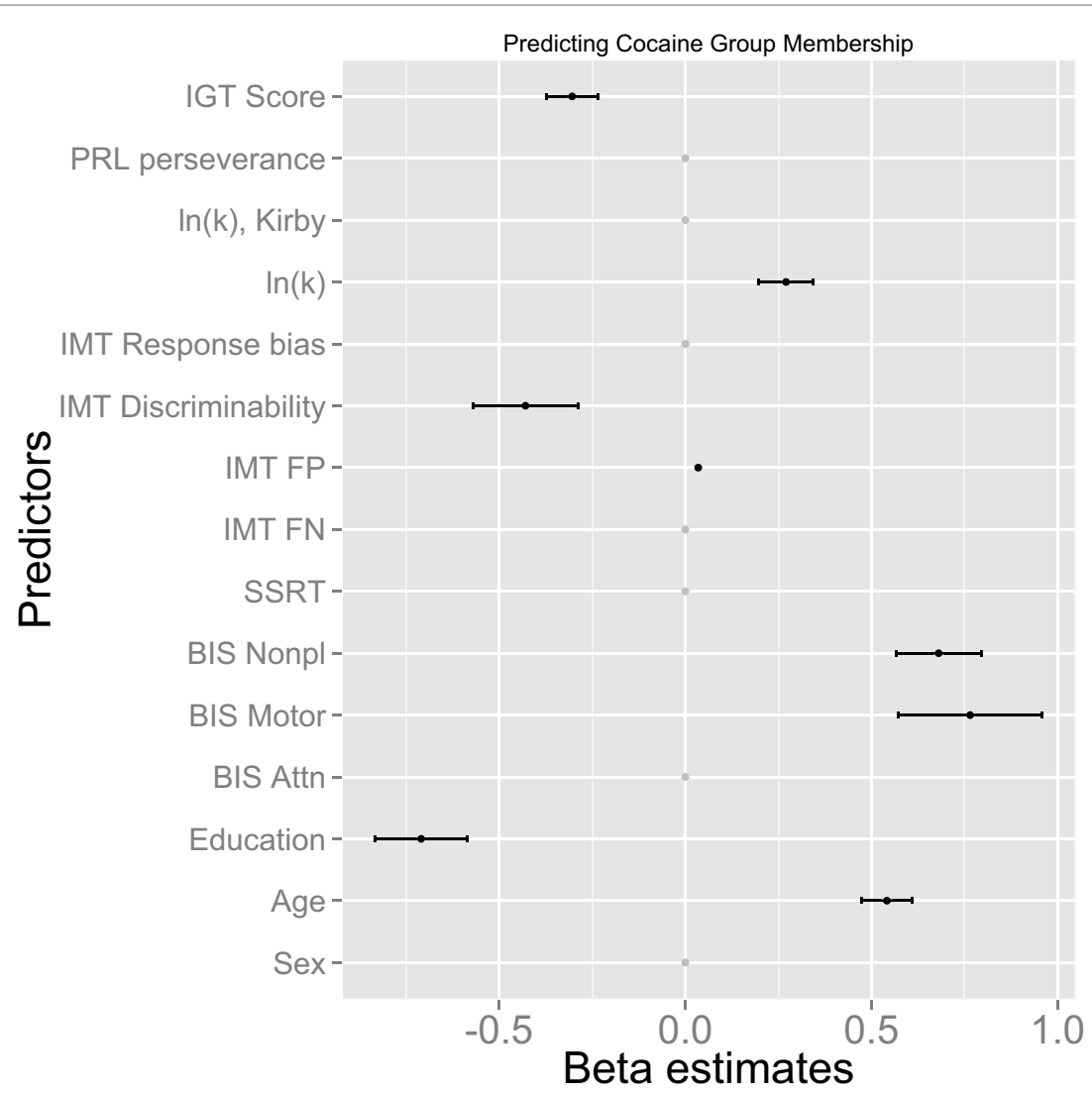

FIGURE 1 | Multivariate patterns of impulsivity measures predicting cocaine dependence. BIS, Barratt Impulsiveness Scale; SSRT, stop-signal reaction time; IMT, Immediate Memory Task; FP, false-positive (commission) errors; FN, false-negative (omission) errors; In(k), natural log of delay-discounting rate; PRL, Probabilistic Reversal Learning; IGT, lowa Gambling Task.

probabilities (0: HC and 1: CDI). A ROC curve was generated by first computing the mean fitted probabilities out of 1,000 iterations in each individual and then using the mean response and actual group information (47).

\section{Robustness of Out-of-Sample Classification Accuracy}

Although our procedure uses separate data for training the LASSO model and testing its out-of-sample classification accuracy, because of our relatively small sample size $(N=54$ in total), one may wonder if the findings will generalize to new samples. To test the robustness of out-of-sample classification accuracy, we permuted the selection of training and test sets 1,000 times (while matching the proportion of CDIs and HCs in each set) and evaluated classification accuracy. In other words, we tested whether we would get similar AUC values when we differently divided data into training and test sets. In each of 1,000 repetitions, we first randomly divided data into training (67\%) and test $(33 \%)$ sets, and then we used the identical procedure (except that the number of iterations of fivefold CV was 100 instead of 1,000) described above to compute the distribution of AUCs on test sets. The data and codes for running the LASSO model are available at https://figshare.com/s/d4b558da3f6af69fc577.

\section{RESULTS}

\section{Subject Characteristics}

Demographic and impulsivity data for all participants are presented in Table 1. The CDIs were older $(t=-4.02 ; p<0.001)$, and reported fewer years of education $(t=5.09 ; p<0.001)$ compared to HCs. CDIs and HCs also differed in their ethnic distribution $\left(\chi^{2}=6.28, p<0.05\right)$. CDIs reported the duration of their regular cocaine use to be $16.0 \pm 9.6$ years. Rates of current regular tobacco use, regular cannabis use, and alcohol dependence in the cocainedependent groups were $74.2,41.9$, and $22.6 \%$, respectively, which is consistent with findings from previous studies (51-53).

\section{Machine-Learning Results}

The training set (67\% of the data) included 21 CDIs and 15 HCs, and the test set (33\% of the data) included 10 CDIs and 8 HCs. Figure 1 shows multivariate patterns of impulsivity and demographic indices predicting $\mathrm{CD}$, revealed by the machinelearning method. Among trait impulsivity measures, higher scores on BIS motor and BIS non-planning impulsivity predicted CD. With respect to impulsive choice, lower IGT net score and higher discounting rate predicted cocaine dependence. On impulsive action, lower IMT discriminability $\left(\mathrm{A}^{\prime}\right)$ and higher 
TABLE 1 | Characteristics of participants with complete data sets for machine-learning analyses.

\begin{tabular}{|c|c|c|c|c|c|c|}
\hline & \multicolumn{2}{|c|}{ Healthy controls $(N=23)$} & \multicolumn{2}{|c|}{ Cocaine users $(N=31)$} & \multirow[t]{2}{*}{ Test statistic } & \multirow[t]{2}{*}{ Sig. } \\
\hline & Mean & SD & Mean & SD & & \\
\hline Age & 35.39 & 12.1 & 47 & 7.78 & -4.02 & 2.89E-04 \\
\hline Sex (\% male) & \multicolumn{2}{|c|}{43.48} & \multicolumn{2}{|c|}{64.52} & 1.59 & n.s. \\
\hline Race (\% W/AA/mixed) & \multicolumn{2}{|c|}{ 26.09/69.56/4.35 } & \multicolumn{2}{|c|}{ 3.23/93.54/3.23 } & 6.28 & 0.043 \\
\hline Education (years) & 15.22 & 2.09 & 12.26 & 2.14 & 5.09 & $5.84 \mathrm{E}-06$ \\
\hline Yrs of cocaine use & 0.00 & 0.00 & 16.02 & 9.56 & -9.32 & $2.28 \mathrm{E}-10$ \\
\hline Yrs of cigarette use & 0.00 & 0.00 & 20.29 & 14.03 & -8.05 & 5.49E-09 \\
\hline Yrs of marijuana use & 0.00 & 0.00 & 12.48 & 12.43 & -5.59 & 4.39E-06 \\
\hline Curr. alcohol abuse (\%) & \multicolumn{2}{|c|}{0.00} & \multicolumn{2}{|c|}{12.90} & 1.60 & n.s. \\
\hline Curr. alcohol dep (\%) & \multicolumn{2}{|c|}{0.00} & \multicolumn{2}{|c|}{22.58} & 4.13 & 0.042 \\
\hline Curr. reg. tobacco use (\%) & \multicolumn{2}{|c|}{0.00} & \multicolumn{2}{|c|}{74.19} & 32.93 & 9.53E-09 \\
\hline Past reg. tobacco use (\%) & \multicolumn{2}{|c|}{0.00} & \multicolumn{2}{|c|}{12.90} & 9.13 & 0.003 \\
\hline Curr. reg. marijuana use (\%) & \multicolumn{2}{|c|}{0.00} & \multicolumn{2}{|c|}{41.94} & 15.10 & $1.02 E-04$ \\
\hline Past reg. marijuana use (\%) & \multicolumn{2}{|c|}{8.70} & \multicolumn{2}{|c|}{29.03} & 6.80 & 0.009 \\
\hline BIS attention & 12.43 & 2.06 & 14.58 & 3.44 & -2.85 & 0.006 \\
\hline BIS motor & 20.48 & 3.29 & 24.35 & 3.96 & -3.92 & 2.61E-04 \\
\hline BIS non-planning & 18.87 & 3.82 & 25.9 & 5.78 & -5.38 & $1.88 \mathrm{E}-06$ \\
\hline SSRT & 293.73 & 86.27 & 316.08 & 84.75 & -0.95 & n.s. \\
\hline IMT omission errors & 14.78 & 8.53 & 16.28 & 10.85 & -0.57 & n.s. \\
\hline IMT commission errors & 20.9 & 12.94 & 35.43 & 16.42 & -3.64 & 0.001 \\
\hline IMT discriminability $\left(A^{\prime}\right)$ & 0.88 & 0.07 & 0.83 & 0.07 & 2.91 & 0.005 \\
\hline IMT response bias $\left(\mathrm{B}_{\mathrm{D}}\right)$ & -0.15 & 0.39 & -0.41 & 0.46 & 2.22 & 0.031 \\
\hline $\ln (k)$ & -6.31 & 1.96 & -4.04 & 2.42 & -3.81 & 3.66E-04 \\
\hline $\ln (k)$, Kirby & -3.92 & 1.35 & -3.07 & 1.44 & -2.24 & 0.030 \\
\hline PRL perseverance errors & 4.74 & 3.26 & 11.55 & 9.70 & -3.64 & 0.001 \\
\hline IGT net score & 23.83 & 32.25 & -4.87 & 26.98 & 3.46 & 0.001 \\
\hline
\end{tabular}

Independent $t$-tests were used to compute significance values, which were uncorrected for multiple comparisons. Regular use is defined as the use of a substance at least 3 days/week for the past 6 months. Curr, current; Reg, regular; W/AA, White/African-American; BIS, Barratt Impulsiveness Scale; SSRT, stop-signal reaction time; IMT, Immediate Memory Task; PRL, Probabilistic Reversal Learning; IGT, lowa Gambling Task; n.s., non-significant ( $p>0.10)$. All tests are t-tests except for sex, race, and \% drug use variables where $\chi^{2}$ tests were used.

IMT commission errors predicted CD. Among demographic variables, lower years of education and higher age predicted CD. Other measures were not critical for the prediction of $\mathrm{CD}$ and their coefficients shrank to 0 .

Figure 2 shows the ROC curve and its mean AUC for the prediction of CD. The AUC was 0.952 for the training set and 0.912 for the test set. Because the age difference between the two groups could be potentially arbitrary, we computed out-ofsample classification accuracy without the age variable in Figure 3 ( $\mathrm{AUC}=0.900$ for the test set).

Note that our out-of-sample classification accuracy was reliably robust (i.e., accuracy was consistently high and above chance) even when we randomly chose training and test sets and computed AUC repeatedly over 1,000 repetitions (see Robustness of Out-of-Sample Classification Accuracy). Figure 4 shows the distribution of AUCs over 1,000 repetitions in training (Figure 4A) and test (Figure 4B) sets. Mean AUC values were 0.974 and 0.887 for the training and test sets, respectively. These results over 1,000 repetitions further increase our confidence that our findings are robust and would be generalizable to new samples. Lastly, we examined classification accuracy with leave-one-out CV [i.e., LASSO is trained on all samples except one and a prediction is made for the left-out participant and the procedure is repeated $N(=54)$ times], where the AUC was even higher (0.976, Figure 5).

\section{DISCUSSION}

This study demonstrates that a multivariate battery of behavioral impulsivity measures can accurately predict CD using machinelearning approaches. The LASSO algorithm selected a subset of measures that were most predictive of CD (BIS motor, BIS nonplanning, IMT commission errors, IMT A', discounting rate of delayed rewards, and IGT net score), while the effects of other measures were 0 due to our use of a shrinkage method (i.e., the LASSO). While some neuroimaging studies have reported moderate to high predictive accuracy for a SUD [e.g., Ref. (33)], to our knowledge, this is one of the first machine-learning studies (32) that achieved such high out-of-sample classification accuracy (AUC $=0.912$ on the test set) using only behavioral measures.

Current findings with behavioral and self-report measures of impulsivity replicate previous findings in cocaine users, suggesting that our task selection was adequate [for a complete review, see Ref. (20)]. Consistent with previous studies $(19,21,22)$ in which CDIs score higher than HCs on motor, non-planning and attentional subscales of the BIS-11 when tested with univariate approaches, the non-planning and motor subscales were most predictive of $\mathrm{CD}$ in the current machine-learning model. On measures of impulsive action, consistent with previous studies $(21,22)$, CDIs had higher commission errors and poorer discriminability compared to controls but did not differ from HCs on omission errors 

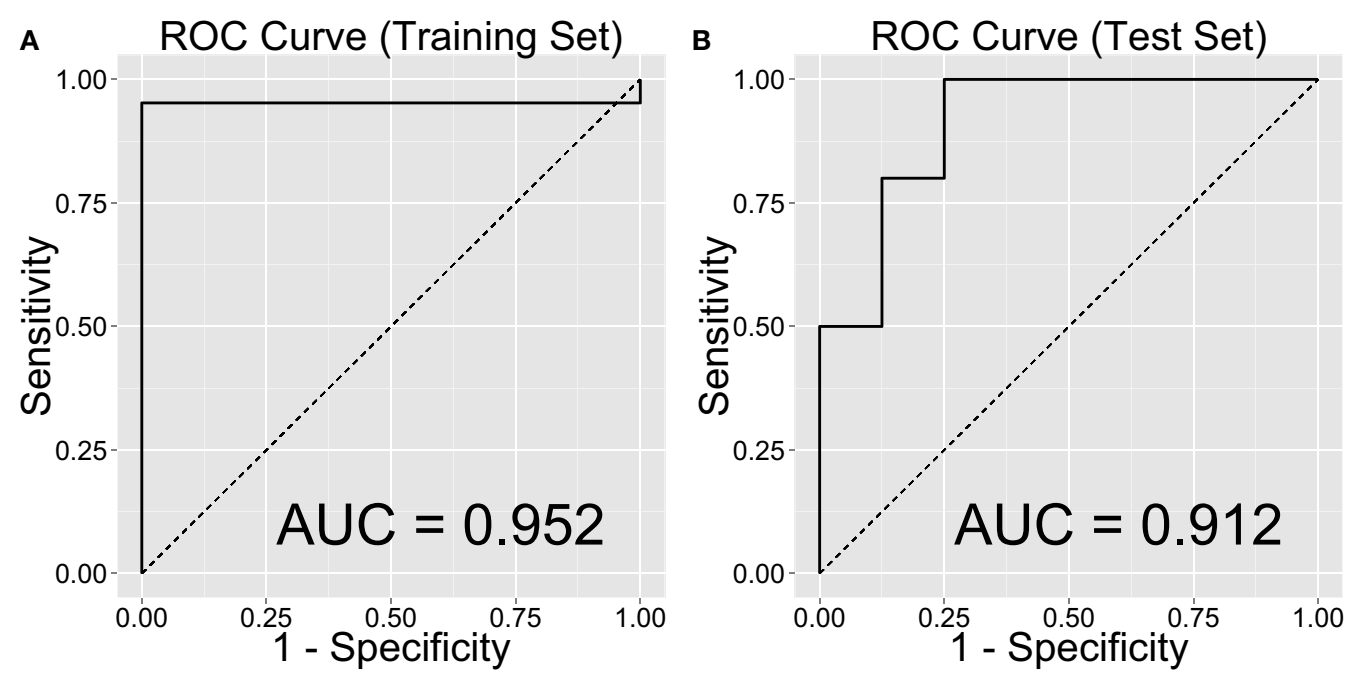

FIGURE 2 | Classification accuracy as indexed by the receiver-operating characteristic (ROC) curves and their area under the curve (AUC), separately on the (A) training and $(B)$ test sets.

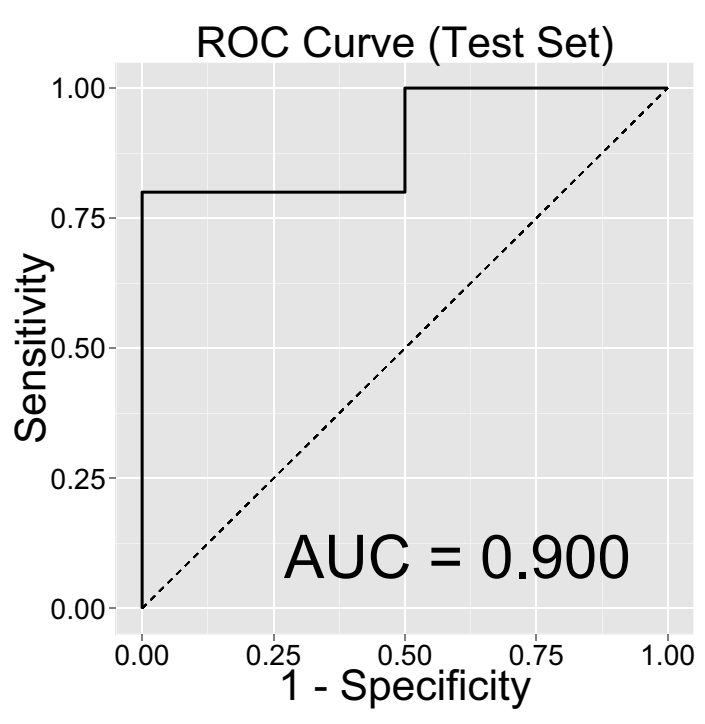

FIGURE 3 | Classification accuracy as indexed by the receiveroperating characteristic $(\mathrm{ROC})$ curve and their area under the curve (AUC) on the test set, after removing the "age" regressor from the LASSO model.

on the IMT. We failed to observe significant group differences on the SST where previous studies have revealed mixed findings (27, 54). With regards to impulsive choice, the literature suggests that CDIs have higher $k$ values on both the Monetary-Choice DelayDiscounting Questionnaire (MCDDT) and Delay-Discounting Task (DDT) compared to HCs $(23,24)$; however, only the DDT task was predictive in our machine-learning model. Consistent with the literature, CDIs also displayed lower net-scores on the IGT $(11,25)$. It is important to note that the LASSO tends to select only one variable if several are correlated to each other. Thus, discrepancy between $t$-test results (Table 1) and machine-learning results could be due to the nature of the LASSO algorithm, which is desirable when the goal is to reduce the number of tasks in the battery as much as possible.

The current study further supports the notion that trait-like and laboratory measures of impulsivity assess non-overlapping and distinct constructs $(11,13,55)$. We used both self-report trait impulsivity and laboratory impulsivity measures (impulsive choice and impulsive action), and as seen in Figure 6, they showed weak to mild correlations (except for within-measure correlations, most absolute values of Pearson correlation coefficients $<0.4$ ). The results suggest that using a battery of impulsivity measures, instead of a single measure, increases prediction accuracy.

Strong predictive markers of SUDs may innovate their prevention and treatment (56), and this study bears important implications for the development of affordable and easy-to-administer standardized assessment batteries that can evaluate individuals' risk to $\mathrm{CD}$ in clinical settings. A rapidly growing literature suggests that there are several neurobiological markers of SUDs, including genetic mutations (57), dopamine D2 receptors levels $(58,59)$, and prefrontal-cortical function $(60,61)$, which serve as vulnerabilities or resilience to SUDs. These neuroimaging and genetic measures, however, are very costly, which makes their use in some clinical settings unsustainable. In contrast, our battery of impulsivity measures (BIS-11, IMT, DDT, and IGT) is cost-effective, brief, and easy to administer, requiring less than an hour to complete. In line with the "precision medicine" initiative (62), this approach may also have utility for personalized personality-targeted prevention and intervention programs [e.g., Ref. (56)], which can be supplemented with various cognitive remediation strategies that address the specific type of impulsivity deficits (action or choice) (63). In another work, we identify distinct substance-specific behavioral profiles for dependence on heroin vs. amphetamines by using a similar machinelearning approach (32). These findings suggest that the efficacy of prevention/intervention programs for SUDs may be improved 

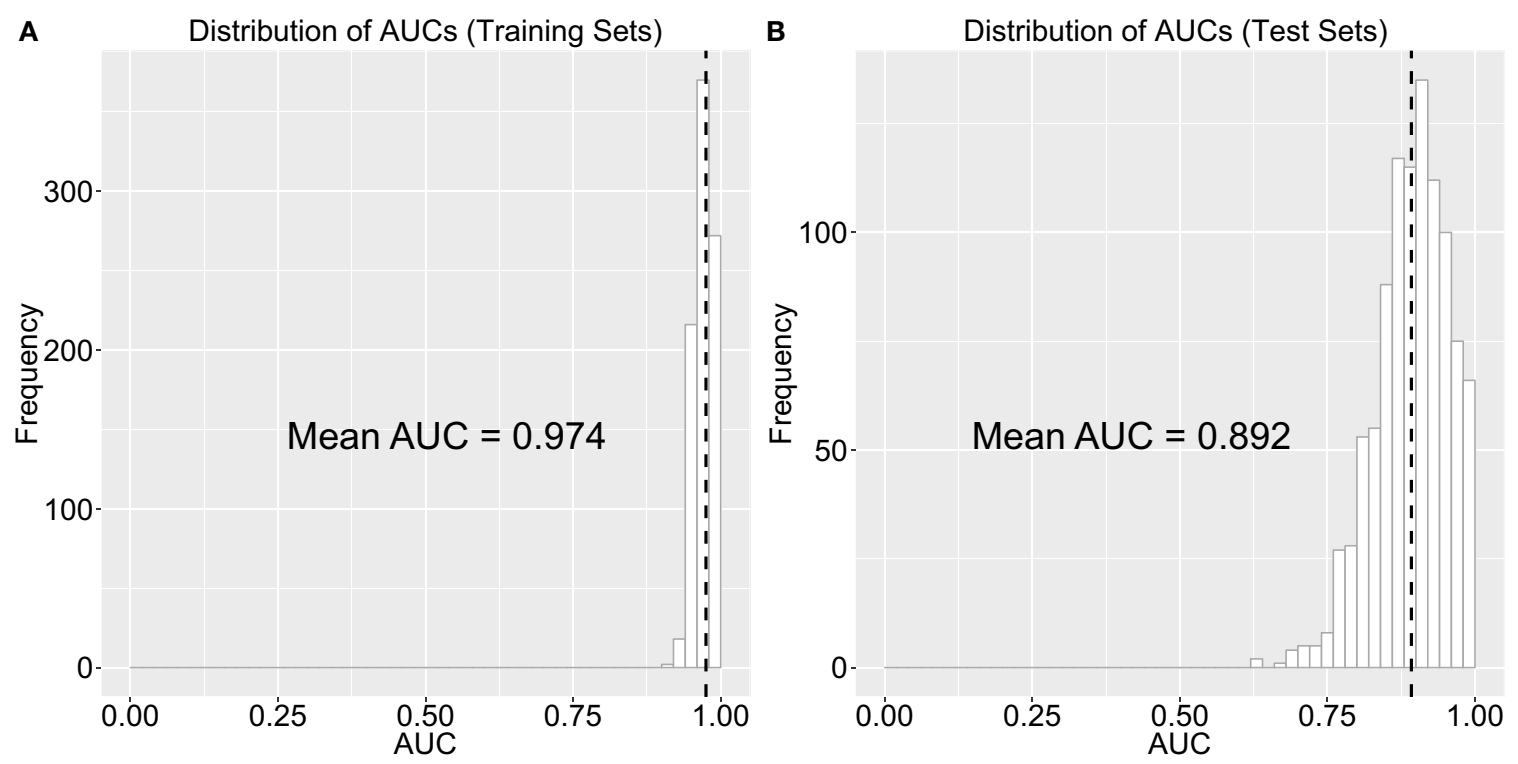

FIGURE 4 | Distribution of the area under the curve (AUC) of the receiver-operating characteristic (ROC) curve on (A) training and (B) test sets when we permutated the selection of training and test sets over $\mathbf{1 , 0 0 0}$ repetitions. The black dotted line indicates the mean AUC of 1,000 repetitions.

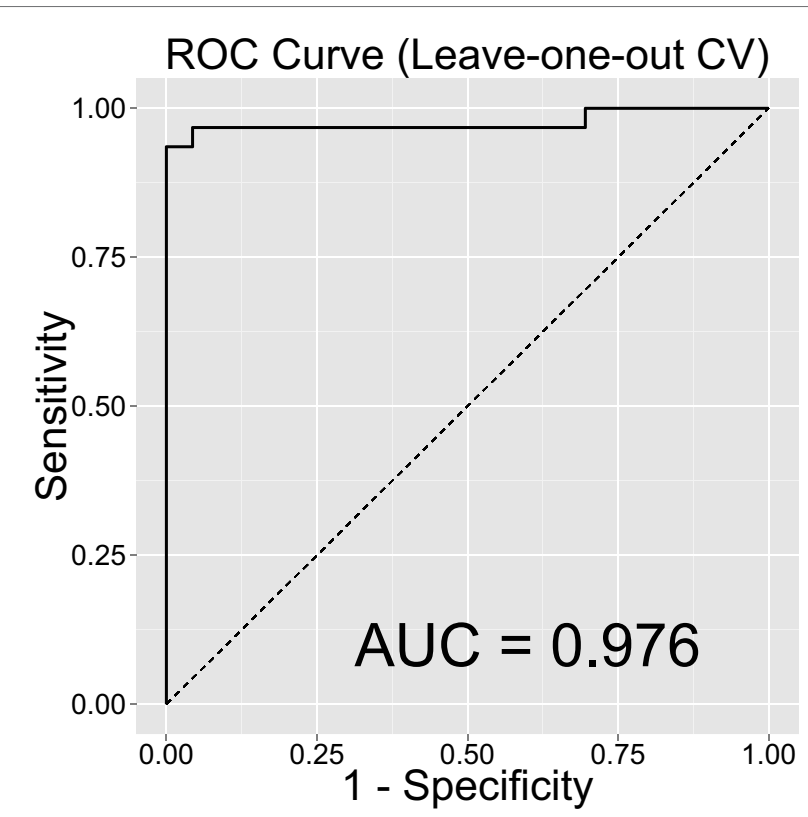

FIGURE 5 | Classification accuracy as indexed by the receiveroperating characteristic (ROC) curves and their area under the curve (AUC) using leave-one-out cross-validation.

by targeting not only individual differences in neurocognitive and personality high-risk profiles but also tailoring preventions/ interventions to dissociable substance-specific high-risk profiles. In line with other studies using a multivariate machine-learning approach (33), some of the strongest predictors of CD in the current study were self-report personality measures, such as the BIS-11. We have previously demonstrated that baseline trait impulsivity (BIS-11) significantly predicts treatment retention and cocaine use among treatment-seeking cocaine users $(19,64)$. We believe the BIS-11 should be considered as a standard measure for studies involving SUDs (36), and we expect our multivariate battery of impulsivity including trait impulsivity measures may be helpful in predicting response to treatment.

In the current study, the predictors of the machine-learning model were all behavioral measures of impulsivity, but ample literature suggests that at least some of them could be linked to biological processes (29): for the IGT, making adaptive choices is related to the function of the prefrontal cortex (PFC), especially its ventromedial part (vmPFC) (65-67). Brain regions related to encoding the subjective values of delayed monetary rewards and choosing delayed rewards have been also well documented $(68,69)$, which include the medial/lateral PFC and ventral striatum. Future studies linking neuroimaging and surrogate measures, such as neurocognitive tasks (70) and eye movements (71), are needed for identifying such surrogate markers of CD.

The current study had several limitations. First, our sample size was relatively small and we acknowledge that larger sample sizes would result in more generalizable findings (72). However, note that out-of-sample classification accuracy was robustly high when we randomly divided data into training and test sets and repeated the procedure. Also, our sample size is greater or comparable to sample sizes in recent neuroimaging studies utilizing machine-learning methods [e.g., Ref. (73-75)]. These neuroimaging studies typically use leave-one-out $\mathrm{CV}$ and have substantially higher number of predictors (i.e., voxels) than our study. Still, we achieved excellent classification accuracy (AUC $=0.912$ on our test set with fivefold CV and AUC $=0.976$ with leave-one-out $\mathrm{CV}$ ), which is higher or comparable to that of neuroimaging studies. Taken together, these results suggest that our findings might generalize in new samples. To our knowledge, 


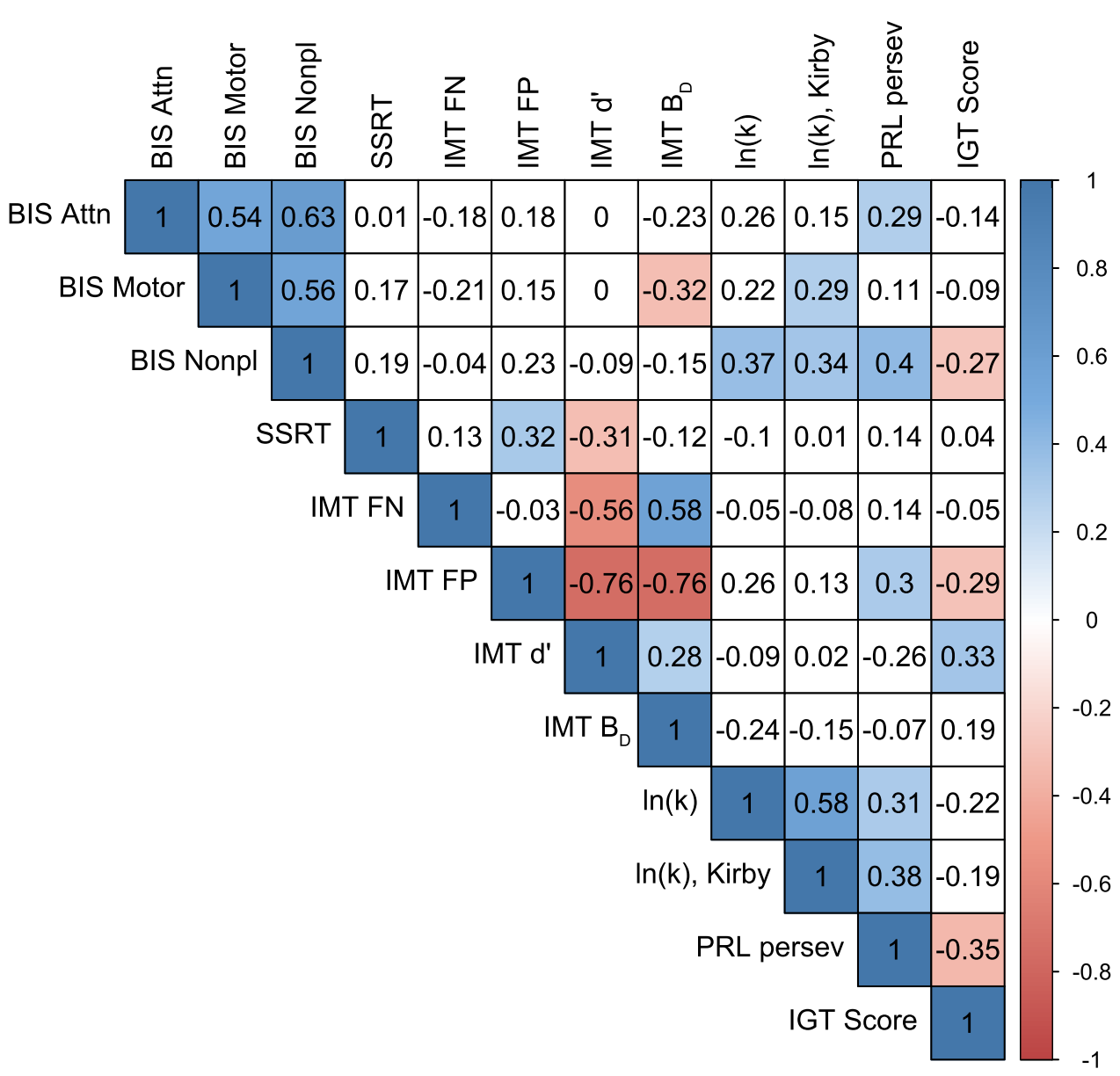

FIGURE 6 | A correlation matrix between all impulsivity measures. Numbers in cells and color bars indicate Pearson correlation coefficients. Significant correlations ( $p<0.05$, uncorrected) are filled with blue (positive correlation) or red (negative correlation) colors. BIS, Barratt Impulsiveness Scale; SSRT, stop-signal reaction time; IMT, Immediate Memory Task; FP, false-positive (commission) errors; FN, false-negative (omission) errors; In(k), natural log of delay-discounting rate; PRL, Probabilistic Reversal Learning; IGT, lowa Gambling Task.

this is one of the first studies applying a machine-learning method for the development of accurate and cost-effective behavioral markers, and the current study might promote awareness and interests in this "big data" approach in the field. The second limitation is that our current participant population abused other substances in addition to cocaine, which is representative of the patterns of drug use in the United States (76), but complicates our attempts to dissociate effects unique to $\mathrm{CD} /$ stimulants from those of other classes of drugs (e.g., opiates) (77). Some of our CDIs also met dependence for substances other than cocaine, including alcohol, nicotine, and marijuana; therefore, our predictors might not be specific to CD in particular. Also, our CDIs are current users, and thus, we are unsure whether their profiles are due to their current state of CD or their preexisting traits related to impulsivity and whether these profiles would persist with abstinence. Lastly, impulse control can be impacted by other conditions including subclinical depression, which we did not measure. However, we believe the impact of depression on our findings would be minimal as we excluded participants meeting DSM-IV criteria for a mood disorder. We address some of these limitations in another study (32), where we recruit individuals in Bulgaria with mono-dependence on heroin or amphetamines who are currently in protracted abstinence.

In summary, the current study demonstrates that a standardized, multivariate behavioral assessment approach to impulsivity, combined with advanced statistical learning approach could advance the field of SUDs in several important ways. With machine-learning algorithms and a multivariate approach, we can refine the design of our studies and improve the prediction accuracy for clinical outcomes. Application of this approach to longitudinal studies might enable us to identify cost-effective behavioral markers for SUDs and identify high-risk neurocognitive or personality profiles (78), which may be targeted independently by prevention/intervention programs.

\section{AUTHOR CONTRIBUTIONS}

W-YA analyzed the data, interpreted the results, and wrote the paper. DR performed research, interpreted the results, and wrote the paper. FM and JV interpreted the results and wrote the 
paper. All authors have approved the final version of the submitted manuscript.

\section{ACKNOWLEDGMENTS}

The authors thank all volunteers for their participation in this study. We thank Warren K. Bickel and Steven Grant for their

\section{REFERENCES}

1. Volkow ND, Skolnick P. New medications for substance use disorders: challenges and opportunities. Neuropsychopharmacology (2012) 37:290-2. doi:10.1038/npp.2011.84

2. Patnode CD, OConnor E, Rowland M, Burda B, Perdue L, Whitlock E. Primary care behavioral interventions to prevent or reduce illicit drug use and nonmedical pharmaceutical use in children and adolescents: a systematic evidence review for the U.S. Preventive Services Task Force. Ann Intern Med (2014) 160:612-20. doi:10.7326/M13-2064

3. Bough KJ, Amur S, Lao G, Hemby SE, Tannu NS, Kampman KM, et al. Biomarkers for the development of new medications for cocaine dependence. Neuropsychopharmacology (2013) 39:202-19. doi:10.1038/ npp.2013.210

4. Fineberg NA, Chamberlain SR, Goudriaan AE, Stein DJ, Vanderschuren LJMJ, Gillan CM, et al. New developments in human neurocognition: clinical, genetic, and brain imaging correlates of impulsivity and compulsivity. CNS Spectr (2014) 19:69-89. doi:10.1017/S1092852913000801

5. Moeller FG, Barratt ES, Dougherty DM, Schmitz JM, Swann AC. Psychiatric aspects of impulsivity. Am J Psychiatry (2001) 158:1783-93. doi:10.1176/appi. ajp.158.11.1783

6. Jentsch JD, Ashenhurst JR, Cervantes MC, Groman SM, James AS, Pennington ZT. Dissecting impulsivity and its relationships to drug addictions. Ann N Y Acad Sci (2014) 1327:1-26. doi:10.1111/nyas.12388

7. Hamilton KR, Littlefield AK, Anastasio NC, Cunningham KA, Fink LHL, Wing VC, et al. Rapid-response impulsivity: definitions, measurement issues, and clinical implications. Personal Disord (2015) 6:168-81. doi:10.1037/ per0000100

8. Hamilton KR, Mitchell MR, Wing VC, Balodis IM, Bickel WK, Fillmore M, et al. Choice impulsivity: definitions, measurement issues, and clinical implications. Personal Disord (2015) 6:182-98. doi:10.1037/per0000099

9. Rangel A, Camerer CF, Montague PR. A framework for studying the neurobiology of value-based decision making. Nat Rev Neurosci (2008) 9:545-56. doi: $10.1038 / \mathrm{nrn} 2357$

10. Cyders MA, Coskunpinar A. Measurement of constructs using self-report and behavioral lab tasks: is there overlap in nomothetic span and construct representation for impulsivity? Clin Psychol Rev (2011) 31:965-82. doi:10.1016/j. cpr.2011.06.001

11. Kjome KL, Lane SD, Schmitz JM, Green C, Ma L, Prasla I, et al. Relationship between impulsivity and decision making in cocaine dependence. Psychiatry Res (2010) 178:299-304. doi:10.1016/j.psychres.2009.11.024

12. Monterosso J, Ehrman R, Napier KL, O’Brien CP, Childress AR. Three decision-making tasks in cocaine-dependent patients: do they measure the same construct? Addiction(2001) 96:1825-37. doi:10.1080/09652140120089571

13. Vassileva J, Paxton J, Moeller FG, Wilson MJ, Bozgunov K, Martin EM, et al. Heroin and amphetamine users display opposite relationships between trait and neurobehavioral dimensions of impulsivity. Addict Behav (2014) 39:652-9. doi:10.1016/j.addbeh.2013.11.020

14. Strakowski SM, Fleck DE, DelBello MP, Adler CM, Shear PK, McElroy SL, et al. Characterizing impulsivity in mania. Bipolar Disord (2009) 11:41-51. doi:10.1111/j.1399-5618.2008.00658.x

15. de Wit H. Impulsivity as a determinant and consequence of drug use: a review of underlying processes. Addict Biol (2008) 14:22-31. doi:10.1111/ j.1369-1600.2008.00129.x

16. Dick DM, Smith G, Olausson P, Mitchell SH, Leeman RF, O’Malley SS, et al. Understanding the construct of impulsivity and its relationship to alcohol use disorders. AddictBiol(2010) 15:217-26. doi:10.1111/j.1369-1600.2009.00190.x

17. Moeller FG, Dougherty DM. Impulsivity and substance abuse: what is the connection? Addict Disord Their Treat (2002) 1:3-10. doi:10.1097/00132576-200205000-00002 helpful comments on the manuscript. Research reported in this publication was supported by the National Institute on Drug Abuse (NIDA) under award number P50DA009262 (FM Co-PI), P50 DA033935 (FM Co-PI), R01DA034131, and VCU CTSA Award UL1TR000058 (FM). W-YA and JV were supported by R01DA021421 (JV) from NIDA and the Fogarty International Center at NIH.

18. Verdejo-García AJ, Perales JC, Pérez-García M. Cognitive impulsivity in cocaine and heroin polysubstance abusers. Addict Behav (2007) 32:950-66 doi:10.1016/j.addbeh.2006.06.032

19. Moeller FG, Dougherty DM, Barratt ES, Schmitz JM, Swann AC, Grabowski J. The impact of impulsivity on cocaine use and retention in treatment. J Subst Abuse Treat (2001) 21:193-8. doi:10.1016/S0740-5472(01)00202-1

20. Spronk DB, van Wel JHP, Ramaekers JG, Verkes RJ. Characterizing the cognitive effects of cocaine: a comprehensive review. Neurosci Biobehav Rev (2013) 37:1838-59. doi:10.1016/j.neubiorev.2013.07.003

21. Moeller FG, Barratt ES, Fischer CJ, Dougherty DM, Reilly EL, Mathias CW, et al. P300 event-related potential amplitude and impulsivity in cocaine-dependent subjects. Neuropsychobiology (2004) 50:167-73. doi:10.1159/000079110

22. Moeller FG, Hasan KM, Steinberg JL, Kramer LA, Dougherty DM, Santos $\mathrm{RM}$, et al. Reduced anterior corpus callosum white matter integrity is related to increased impulsivity and reduced discriminability in cocaine-dependent subjects: diffusion tensor imaging. Neuropsychopharmacology (2005) 30:610-7. doi:10.1038/sj.npp.1300617

23. Heil SH, Johnson MW, Higgins ST, Bickel WK. Delay discounting in currently using and currently abstinent cocaine-dependent outpatients and non-drugusing matched controls. Addict Behav (2006) 31:1290-4. doi:10.1016/j. addbeh.2005.09.005

24. Kirby KN, Petry NM. Heroin and cocaine abusers have higher discount rates for delayed rewards than alcoholics or non-drug-using controls. Addiction (2004) 99:461-71. doi:10.1111/j.1360-0443.2003.00669.x

25. Bolla KI, Eldreth DA, London ED, Kiehl KA, Mouratidis M, Contoreggi C, et al. Orbitofrontal cortex dysfunction in abstinent cocaine abusers performing a decision-making task. Neuroimage (2003) 19:1085-94. doi:10.1016/ S1053-8119(03)00113-7

26. Ersche KD, Roiser JP, Robbins TW, Sahakian BJ. Chronic cocaine but not chronic amphetamine use is associated with perseverative responding in humans. Psychopharmacology (2008) 197:421-31. doi:10.1007/s00213-007-1051-1

27. Fillmore MT, Rush CR. Impaired inhibitory control of behavior in chronic cocaine users. Drug Alcohol Depend (2002) 66:265-73. doi:10.1016/ S0376-8716(01)00206-X

28. Li C-SR, Milivojevic V, Kemp K, Hong K, Sinha R. Performance monitoring and stop signal inhibition in abstinent patients with cocaine dependence. Drug Alcohol Depend (2006) 85:205-12. doi:10.1016/j.drugalcdep.2006.04.008

29. Volkow ND, Koob G, Baler R. Biomarkers in substance use disorders. ACS Chem Neurosci (2015) 6:522-5. doi:10.1021/acschemneuro.5b00067

30. Hastie TJ, Tibshirani RJ, Friedman JJH. The Elements of Statistical Learning. New York, NY: Springer (2009).

31. Whelan R, Garavan H. When optimism hurts: inflated predictions in psychiatric neuroimaging. Biol Psychiatry (2014) 75:746-8. doi:10.1016/j. biopsych.2013.05.014

32. Ahn W-Y, Vassileva J. Machine-learning identifies substance-specific behavioral markers for opiate and stimulant dependence. Drug Alcohol Depend (in press). doi:10.1016/j.drugalcdep.2016.02.008

33. Whelan R, Watts R, Orr CA, Althoff RR, Artiges E, Banaschewski T, et al. Neuropsychosocial profiles of current and future adolescent alcohol misusers. Nature (2014) 512:185-9. doi:10.1038/nature13402

34. First MB, Gibbon M. User's Guide for the Structured Clinical Interview for DSM-IV Axis I Disorders. Washington, DC: American Psychiatric Pub (1997).

35. Patton JH, Stanford MS, Barratt ES. Factor structure of the Barratt impulsiveness scale. JClin Psychol (1995) 51:768-74. doi:10.1002/1097-4679(199511)51:6<768:aid-jclp2270510607>3.0.co;2-1

36. Stanford MS, Mathias CW, Dougherty DM. Fifty years of the Barratt impulsiveness scale: an update and review. Pers Individ Dif (2009) 47:385-95. doi:10.1016/j.paid.2009.04.008

37. Dougherty DM, Marsh DM, Mathias CW. Immediate and delayed memory tasks: a computerized behavioral measure of memory, attention, and 
impulsivity. Behav Res Methods Instrum Comput (2002) 34:391-8. doi:10.3758/ BF03195467

38. Donaldson W. Measuring recognition memory. J Exp Psychol Gen (1992) 121:275-7. doi:10.1037/0096-3445.121.3.275

39. Logan GD, Cowan WB. On the ability to inhibit thought and action: a theory of an act of control. Psychol Rev (1984) 91:295-233. doi:10.1037/0033-295X.91.3.295

40. Verbruggen F, Logan GD. Response inhibition in the stop-signal paradigm. Trends Cogn Sci (2008) 12:418-24. doi:10.1016/j.tics.2008.07.005

41. Johnson MW, Bickel WK. Within-subject comparison of real and hypothetical money rewards in delay discounting. J Exp Anal Behav (2002) 77:129-46. doi:10.1901/jeab.2002.77-129

42. Kirby KN, Petry NM, Bickel WK. Heroin addicts have higher discount rates for delayed rewards than non-drug-using controls. J Exp Psychol Gen (1999) 128:78. doi:10.1037/0096-3445.128.1.78

43. Wileyto EP, Audrain-McGovern J, Epstein LH, Lerman C. Using logistic regression to estimate delay-discounting functions. Behav Res Methods Instrum Comput (2004) 36:41-51. doi:10.3758/BF03195548

44. Bechara A, Dolan S, Denburg N, Hindes A, Anderson SW, Nathan PE. Decision-making deficits, linked to a dysfunctional ventromedial prefrontal cortex, revealed in alcohol and stimulant abusers. Neuropsychologia (2001) 39:376-89. doi:10.1016/S0028-3932(00)00136-6

45. Cools R, Clark L, Owen AM, Robbins TW. Defining the neural mechanisms of probabilistic reversal learning using event-related functional magnetic resonance imaging. J Neurosci (2002) 22(11):4563-7.

46. Tibshirani R. Regression shrinkage and selection via the lasso. J R Stat Soc Ser B Stat Methodol (1996) 58(1):267-88.

47. Ahn W-Y, Kishida KT, Gu X, Lohrenz T, Harvey A, Alford JR, et al. Nonpolitical images evoke neural predictors of political ideology. Curr Biol (2014) 24:2693-9. doi:10.1016/j.cub.2014.09.050

48. Hastie T. Regularization paths for generalized linear models via coordinate descent (2010) 33:1. Available from: http://www.ncbi.nlm.nih.gov/pmc/ articles/PMC2929880/

49. Robin X, Turck N, Hainard A, Tiberti N, Lisacek F, Sanchez J-C, et al. pROC: an open-source package for R and S+ to analyze and compare ROC curves. BMC Bioinformatics (2011) 12:77. doi:10.1186/1471-2105-12-77

50. Hosmer DW, Lemeshow S. Applied Logistic Regression. Hoboken, NJ: John Wiley \& Sons (2004).

51. Lindsay JA, Stotts AL, Green CE, Herin DV, Schmitz JM. Cocaine dependence and concurrent marijuana use: a comparison of clinical characteristics. Am J Drug Alcohol Abuse (2009) 35:193-8. doi:10.1080/ 00952990902933860

52. Liu S, Lane SD, Schmitz JM, Waters AJ, Cunningham KA, Moeller FG. Relationship between attentional bias to cocaine-related stimuli and impulsivity in cocaine-dependent subjects. Am J Drug Alcohol Abuse (2011) 37:117-22. doi:10.3109/00952990.2010.543204

53. Ramesh D, Keyser-Marcus LA, Ma L, Schmitz JM, Lane SD, Marwitz JH, et al. Prevalence of traumatic brain injury in cocaine-dependent research volunteers. Am J Addict (2015) 24(4):341-7. doi:10.1111/ajad.12192

54. Li C-SR, Huang C, Yan P, Bhagwagar Z, Milivojevic V, Sinha R. Neural correlates of impulse control during stop signal inhibition in cocainedependent men. Neuropsychopharmacology (2008) 33:1798-806. doi:10.1038/ sj.npp. 1301568

55. Caswell AJ, Bond R, Duka T, Morgan MJ. Further evidence of the heterogeneous nature of impulsivity. Pers Individ Dif (2015) 76:68-74. doi:10.1016/j. paid.2014.11.059

56. Conrod PJ, Castellanos-Ryan N, Strang J. Brief, personality-targeted coping skills interventions and survival as a non-drug user over a 2-year period during adolescence. Arch Gen Psychiatry (2010) 67:85-93. doi:10.1001/ archgenpsychiatry.2009.173

57. Ducci F, Goldman D. The genetic basis of addictive disorders. Psychiatr Clin North Am (2012) 35:495-519. doi:10.1016/j.psc.2012.03.010

58. Volkow ND, Wang G-J, Begleiter H, Porjesz B, Fowler JS, Telang F, et al. High levels of dopamine $\mathrm{d} 2$ receptors in unaffected members of alcoholic families: possible protective factors. Arch Gen Psychiatry (2006) 63:999-1008. doi:10.1001/archpsyc.63.9.999

59. Volkow ND, Fowler JS, Wang GJ, Baler R, Telang F. Imaging dopamine's role in drug abuse and addiction. Neuropharmacology (2009) 56(Suppl 1):3-8. doi:10.1016/j.neuropharm.2008.05.022
60. Goldstein RZ, Volkow ND. Dysfunction of the prefrontal cortex in addiction: neuroimaging findings and clinical implications. Nat Rev Neurosci (2011) 12:652-69. doi:10.1038/nrn3119

61. Volkow ND, Baler RD. Addiction science: uncovering neurobiological complexity. Neuropharmacology (2014) 76:235-49. doi:10.1016/j. neuropharm.2013.05.007

62. Collins FS, Varmus H. A new initiative on precision medicine. $N$ Engl J Med (2015) 372:793-5. doi:10.1056/NEJMp1500523

63. Bickel WK, Yi R, Landes RD, Hill PF, Baxter C. Remember the future: working memory training decreases delay discounting among stimulant addicts. Biol Psychiatry (2011) 69:260-5. doi:10.1016/j.biopsych.2010.08.017

64. Hulka LM, Vonmoos M, Preller KH, Baumgartner MR, Seifritz E, Gamma A, et al. Changes in cocaine consumption are associated with fluctuations in self-reported impulsivity and gambling decision-making. Psychol Med (2015) 45:3097-110. doi:10.1017/S0033291715001063

65. Ahn W-Y, Krawitz A, Kim W, Busemeyer JR, Brown JW. A model-based fMRI analysis with hierarchical Bayesian parameter estimation. J Neurosci Psychol Econ (2011) 4:95-110. doi:10.1037/a0020684

66. Bechara A, Damasio AR, Damasio H, Anderson SW. Insensitivity to future consequences following damage to human prefrontal cortex. Cognition (1994) 50:7-15. doi:10.1016/0010-0277(94)90018-3

67. Li X, Lu Z-L, D'Argembeau A, Ng M, Bechara A. The Iowa gambling task in fMRI images. Hum Brain Mapp (2009) 31(3):410-23. doi:10.1002/hbm.20875

68. Kable JW, Glimcher PW. The neural correlates of subjective value during intertemporal choice. Nat Neurosci (2007) 10:1625-33. doi:10.1038/nn2007

69. McClure SM, Laibson DI, Loewenstein G, Cohen JD. Separate neural systems value immediate and delayed monetary rewards. Science (2004) 306:503-7. doi:10.1126/science.1100907

70. Evans DE, Park JY, Maxfield N, Drobes DJ. Neurocognitive variation in smoking behavior and withdrawal: genetic and affective moderators. Genes Brain Behav (2009) 8:86-96. doi:10.1111/j.1601-183X.2008.00445.x

71. Groman SM, James AS, Seu E, Tran S, Clark TA, Harpster SN, et al. In the blink of an eye: relating positive-feedback sensitivity to striatal dopamine D2-like receptors through blink rate. J Neurosci (2014) 34:14443-54. doi:10.1523/ JNEUROSCI.3037-14.2014

72. Button KS, Ioannidis JPA, Mokrysz C, Nosek BA, Flint J, Robinson ESJ, et al. Power failure: why small sample size undermines the reliability of neuroscience. Nat Rev Neurosci (2013) 14:365-76. doi:10.1038/nrn3475

73. Hoeft F, McCandliss BD, Black JM, Gantman A, Zakerani N, Hulme C, et al. Neural systems predicting long-term outcome in dyslexia. Proc Natl Acad Sci U S A (2011) 108:361-6. doi:10.1073/pnas.1008950108

74. Lavagnino L, Amianto F, Mwangi B, D’Agata F, Spalatro A, Zunta-Soares GB, et al. Identifying neuroanatomical signatures of anorexia nervosa: a multivariate machine learning approach. Psychol Med (2015) 45:2805-12. doi:10.1017/ S0033291715000768

75. Ullman H, Almeida R, Klingberg T. Structural maturation and brain activity predict future working memory capacity during childhood development. J Neurosci (2014) 34:1592-8. doi:10.1523/JNEUROSCI.0842-13.2014

76. Fernandez-Serrano MJ, Pérez-García M, Verdejo-García A. What are the specific vs. generalized effects of drugs of abuse on neuropsychological performance? Neurosci Biobehav Rev (2011) 35:377-406. doi:10.1016/j. neubiorev.2010.04.008

77. Badiani A, Belin D, Epstein D, Calu D, Shaham Y. Opiate versus psychostimulant addiction: the differences do matter. Nat Rev Neurosci (2011) 12:685-700. doi:10.1038/nrn3104

78. Ahn W-Y, Busemeyer JR. Challenges and promises for translating computational tools into clinical practice. Curr Opin Behav Sci (in press). doi:10.1016/j.cobeha.2016.02.001

Conflict of Interest Statement: The authors declare that the research was conducted in the absence of any commercial or financial relationships that could be construed as a potential conflict of interest.

Copyright (c) 2016 Ahn, Ramesh, Moeller and Vassileva. This is an open-access article distributed under the terms of the Creative Commons Attribution License (CC BY). The use, distribution or reproduction in other forums is permitted, provided the original author(s) or licensor are credited and that the original publication in this journal is cited, in accordance with accepted academic practice. No use, distribution or reproduction is permitted which does not comply with these terms. 